\title{
How Does the Mindfulness-Based Program for Infertility (MBPI) Work in Reducing Depressive Symptoms?
}

\author{
Ana Galhardo ${ }^{1,2}$ (i) $\cdot$ Mariana Moura-Ramos ${ }^{2}$ Marina Cunha ${ }^{1,2} \cdot$ José Pinto-Gouveia $^{2}$
}

(C) Springer Science+Business Media, LLC 2017

\begin{abstract}
Mindfulness-based approaches have been applied and proved efficient in reducing symptoms related to several health problems. To our knowledge, the Mindfulness-Based Program for Infertility (MBPI) was the first mindfulness program developed specifically to target women facing infertility. This psychological intervention program was previously shown to be effective in reducing depressive symptoms in women facing infertility, but the underlying mechanisms were not clarified. The current study aimed to test whether the decrease of depressive symptoms could be explained by the increase of self-efficacy following the MBPI. The MBPI included 10 weekly sessions, in a group format, with the duration of about $2 \mathrm{~h}$ each and was applied to five groups (10 to 15 participants in each group). Fifty-five women completed the MBPI, and 37 women were assigned to a control group. All participants presented an infertility diagnosis and were pursuing medical treatment for this condition. Standardized measures of depression and infertility self-efficacy were collected pre and post MBPI. A significant indirect effect was found, showing that the MBPI intervention reduced the depressive symptoms of women with infertility by increasing their perceptions of self-efficacy. In summary, the promotion of infertility self-efficacy may be considered a key component of psychological interventions targeting depressive symptoms in women facing infertility.
\end{abstract}

Ana Galhardo

anagalhardo@ismt.pt

1 Instituto Superior Miguel Torga, Largo da Cruz de Celas, ${ }^{\circ} 1$, 3000-132 Coimbra, Portugal

2 CINEICC - Faculty of Psychology and Educational Sciences, University of Coimbra, R. do Colégio Novo, 10A,

3001-802 Coimbra, Portugal
Keywords Infertility · Mindfulness-Based Program for Infertility (MBPI) - Infertility self-efficacy · Depressive symptoms

\section{Introduction}

Infertility is defined by the International Committee for Monitoring Assisted Reproductive Technology (ICMART) and the World Health Organization as the "failure to achieve a pregnancy after 12 months or more of regular unprotected sexual intercourse" (Zegers-Hochschild et al. 2009). Furthermore, it can also be considered as a social and emotional condition described as a low-control stressor in which the couple is challenged with the unfulfilled goal of parenthood (Greil 1997). Although prevalence estimates of lifetime infertility vary, a systematic analysis of 277 health surveys estimated that 48.5 million couples worldwide suffer from infertility (Mascarenhas et al. 2012). More recently, Datta et al. (2016) stated that one in eight women and one in 10 men aged 16-74 years had experienced infertility and from these $57.3 \%$ of women and $53.2 \%$ of men had sought medical or professional help in order to solve fertility problems.

Dealing with infertility is frequently perceived as a physically and psychologically challenging experience. In this context, Covington and Adamson (2015) mentioned that feelings of defectiveness, inadequacy, inferiority, worthlessness, shame, and guilt are commonly experienced. Studies addressing psychological consequences of infertility have shown inconclusive results. Chen et al. (2004) indicated that women pursuing infertility medical treatment exhibit a high prevalence of psychiatric disorders, such as generalized anxiety disorder (23.2\%) and major depression (17.0\%). In a study conducted by Volgsten et al. (2010), 30.8\% of women and $10.2 \%$ of men undergoing in vitro fertilization (IVF) presented 
with a psychiatric diagnosis with major depression being the most common mood disorder. Furthermore, in a register-based national cohort study, Sejbaek et al. (2013) found that women diagnosed with depression prior to assisted reproduction technology (ART) treatment started significantly fewer treatment cycles and had a lower mean number of live births compared to women without a psychiatric history of depression. Contrastingly, Greil (1997) and Eugster and Vingerhoets (1999) emphasized more similarities than differences between people with an infertility diagnosis and comparison groups. Moreover, in a systematic review, Verhaak et al. (2007) pointed to only minor differences in emotional difficulties when comparing women starting IVF and a control group.

Despite the differences in these reported findings, people facing infertility experience emotional distress that can vary in terms of intensity and duration. Most of them tend to adjust well psychologically, but some may experience depression and anxiety symptoms with clinical significance (MouraRamos et al. 2010; Verhaak et al. 2010).

It is hypothesized that one of the constructs that seems to be related to these depressive symptoms is self-efficacy. In fact, the association between self-efficacy and depression in people facing infertility has been established (Faramarzi et al. 2014; Fu et al. 2016; Galhardo et al. 2013a) being postulated that women who perceive themselves as having confidence and skills to deal with the diagnosis of infertility and the demands of medical treatment would be less prone to show depressive symptoms.

According to Bandura (1994), self-efficacy corresponds to beliefs people have about their own abilities to accomplish goals. When it comes to health, self-efficacy can be defined as a psychosocial concept that encompasses a person's capability to care for his/her own health and to overcome illness (Gandoy-Crego et al. 2016).

In the specific area of infertility, Cousineau et al. (2006) stated that self-efficacy comprises several self-regulation processes (cognitive, affective, and motivational) and influences the ability to deal with various circumstances. Moreover, these authors highlighted that demonstrating high self-efficacy to deal with infertility tends to be associated to more positive emotional states, a higher persistence in pursuing medical treatment, and a more open attitude towards other familybuilding options. Additionally, mindfulness has been associated with several different forms of self-efficacy (e.g., Hou et al. 2014; Turner et al. 2016) and greater use of mindfulness skills has been associated with greater coping self-efficacy, which in turn partially mediated the relationship between each of these skills and emotion regulation difficulties (Luberto et al. 2014).

Mindfulness-based interventions have been applied to several health problems such as chronic pain, cancer, anxiety disorders, and depression, and evidence regarding their efficacy has been achieved (Baer 2003; Gotink et al. 2015). The
Mindfulness-Based Program for Infertility (MBPI) (Galhardo et al. 2013b) is based on the Mindfulness-Based Program for Stress Reduction (Kabat-Zinn 1990), the Mind Body Program for Infertility (Domar et al. 2000), and basic principles of Acceptance and Commitment Therapy (Hayes et al. 1999) and was developed specifically for targeting people dealing with fertility problems. The MBPI was intended to develop mindfulness and acceptance skills and to promote the perception of self-efficacy to deal with the infertility diagnosis and constrains of medical treatment. The cultivation of awareness through mindfulness practice might be a way of being in touch in an open and non-judgmental way, moment by moment, with painful inner mental states (e.g., feelings, thoughts, and memories). In addition, attitudes of kindness, curiosity, and willingness to be present with the unfolding experience are promoted as well as the ability to recognize one's experiences as part of the larger human experience. Another key component of the MBPI is clarification and the promotion of value-congruent living. Clarifying values or chosen life directions allows defining what gives one's life a sense of meaning or purpose and to use values as a guide for one's actions (Harris 2009). Furthermore, being able to recognize one's private events (e.g., thoughts, emotions, bodily sensations) just as they are enables us to choose and engage in adequate actions to respond to the situations, providing a sense of selfefficacy (Williams et al. 2007).

The MBPI encompassed 10 weekly 2-h sessions and was delivered in a group format to five groups ( 55 participants) by the first author who is a clinical psychologist with mindfulness training and experience. Therapist and participant's manuals were available, and audio files with mindfulness instructions were provided to participants in order to support mindfulness practice between sessions. The Therapist Manual included a detailed description of each session contents and experiential exercises to be accomplished. Through a multimodal range of mindfulness and acceptance exercises, participants were provided with tools to become more conscious and tolerant of their moment to moment experiences, and learn new ways of soothing themselves in face of emotional distress. MBPI also addressed values clarification in order to increase the adoption of actions consistent with one's life values. Being able to act in a meaningful way was expected to promote self-efficacy. Between-session assignments (e.g., daily selfmonitoring, mindfulness and self-compassion exercises, and gratitude exercises) were recommended to allow participants practicing these skills as well as integrating new ways of relating to their internal and external experiences. Furthermore, the group format, the inclusion of sharing, and a psychoeducational component addressing common psychological consequences of dealing with infertility and healthy lifestyle (exercise, nutrition, caffeine, alcohol, nicotine, etc.) were intended to promote a sense of common humanity (see Galhardo et al. 2013b for further details). 
This psychological group intervention program was previously shown to be effective in reducing depressive symptoms, internal and external shame, and entrapment and defeat, in women with an infertility diagnosis pursuing medical treatment for their fertility issues (Galhardo et al. 2013b). Inversely, it led to a significant improvement in mindfulness skills and to an increase in participants' sense of self-efficacy to deal with infertility (Galhardo et al. 2013b). Moreover, the MBPI was shown to be feasible and well accepted by partic(Galhardo et al. 2016). Though the efficacy study was not a randomized controlled trial, participants were equally interested in completing the program and they did not differ in terms of demographic and study variables at baseline. Although the MBPI showed promising results, the underlying mechanisms of its effectiveness need further clarification. Indeed, it may be hypothesized that the effect of the MBPI in reducing psychological symptoms may not be a direct effect, but an indirect effect throughout the development of new skills, such as perceptions of self-efficacy.

Results from the MBPI indicated that participants significantly increased their perception of infertility self-efficacy, but the specific role of self-efficacy as a mediator was not explored in the previously conducted study on the efficacy of the MBPI. As such and considering that changes in the way people with infertility perceive their ability to face this diagnosis and the strains of medical treatment may influence their mental health status, the aim of the current study was to explore the mechanism by which the MBPI helps reduce depressive symptoms. More specifically, based on the previous titit was hypothesized that the decrease of depressive symptoms in women who attended the MBPI would be influenced by the increase of their self-efficacy in dealing with infertility issues.

\section{Method \\ Participants}

A total of 102 women answered a recruitment announcement posted at the Portuguese Fertility Association (patients association) website presenting the study aims. Inclusion criteria were age (18 years or older) and a medical diagnosis of infertility. Participants were all married or living with a partner in a heterosexual relationship (as per requirements of the Portuguese law for the access to ART at time of data collection). The MBPI was applied to a total of 55 women who took part in five separate groups. Control group participants $(n=37)$ were recruited through the same procedure, but were invited to take part in the control group as they would not be able to attend the weekly group sessions due to constrains. The MBPI sessions were held between May 2009 and May 2010.

\section{Procedure}

The study was approved by the Scientific Committee of the Faculty of Psychology and Educational Sciences of the University of Coimbra, Portugal. The national patients association supported participants' recruitment. The aims of the study, inclusion criteria, participants' role, researchers' obligations, and procedure to participate (contact the first author by email) were explained in the recruitment post placed on the national patients' association website. Women in the control group (waitlist) were those who answered this post but did not live in the cities where the study took place and thus could not attend the MBPI sessions (they agreed to join the control group and were given the opportunity to participate in future sessions). Participants in both groups were pursuing infertility medical treatment at the same clinics, but travel expenses to attend the weekly MBPI sessions would add financial burden to the ones living in other cities. Participants provided infertility clinical information (there was no consulting of medical records). All the subjects gave their written informed consent. The study had a longitudinal design. A set of self-report measures was completed by the MBPI group at baseline and dur the first week following the last MBPI session. Besides the self-report instruments, participants in the MBPI group also completed a semi-structured clinical interview adapted from the admission interview of the Mind Body Program for Infertility (Domar et al. 1990) prior to taking part in the MBPI groups. This approximately 1-h-duration interview was conducted by the first author and addresses demographic and clinical data and allows the screening for severe psychopathology. In the control group, the assessment protocol was sent and returned by mail (stationary post envelopes were provided). Participants enrolled in the control group completed the self-report measures at the same time points of the MBPI group participants. No attrition bias occurred from baseline to post-MBPI in the MBPI or the control group.

\section{Measures}

Attending to the aims of the current study, the following selfreport measures were used:

The Beck Depression Inventory (BDI-Beck et al. 1961; Portuguese version by Vaz-Serra and Abreu 1973) is a widely used self-report measure of depressive symptoms that encompasses 21 groups of statements. Subjects were asked to indicate which statement best represented their experience. The BDI is an extensively accepted instrument for assessing depression in clinical samples and detecting depression in erat populations. In the current study, Cronbach's alpha coefficient for the BDI was 0.85 .

The Infertility Self-Efficacy Scale (ISE; Cousineau et al. 2006), Portuguese version by Galhardo et al. (2013a), is a 16-item self-report instrument assessing the perception of 
capability to use ones' cognitive, emotional, and behavioral abilities for dealing with the demands of an infertility diagnosis and medical treatment. Subjects are asked on a 9-point scale to indicate the degree to which they feel confident regarding tasks such as "Make meaning out of my infertility experience," "Accept that my best efforts may not change my/our infertility," and "Keep active with my usual life routine." In this study, the ISE Cronbach alpha value was 0.95 .

\section{Data Analyses}

Data were analyzed using SPSS and PROCESS macro for SPSS developed by Hayes (2013). Firstly, we conducted an ANOVA for repeated measures, to examine the interaction effects between Time [within-subject factor $(\mathrm{T} 1=$ pre-treatment and $\mathrm{T} 2=$ post-treatment)] and Group (between-subject factor; $0=$ control; $1=$ MBPI) for self-efficacy and depressive symptoms scores. With these analyses, differences in changes between pre- and post-treatment scores across the two groups were documented. Secondly, the mechanism of change in these scores was tested.

The analytic procedure was conducted according to Hayes and Rockwood's (2016) recommendations. To understand the mechanism underlying the effect of the MBPI on the reduction of depressive symptoms scores, we conducted a mediation analysis based upon regression analysis. In this model, the intervention was the predictor (coded as $0=$ control; $1=\mathrm{MBPI}$ ), self-efficacy at $\mathrm{T} 2$ was the mediator variable, and depressive symptoms at $\mathrm{T} 2$ was the outcome variable. As suggested by Hayes and Rockwood (2016), self-efficacy and depressive symptoms at $\mathrm{T} 1$ were included as covariates in the model, to allow for a better prediction model, rather than using computed variables based on the changes in these scores. A mediation model was conducted to evaluate indirect and direct effects using PROCESS macro in SPSS (Model 4) with bootstrap procedures (5000 samples), as recommended by Mackinnon et al. (2004). A confidence interval of $95 \%$ was calculated in all the analyses.

\section{Results}

Participants' descriptive statistics are reported in Table 1. The MPBI group and the control group were considered to be equivalents concerning sociodemographic variables (years of education, marital status, and years of marriage).

There was a significant difference between the groups regarding age ( $p=.049 ; d=0.42$ ). No differences were found regarding clinical variables (infertility duration and infertility diagnosis). Regarding group comparisons at baseline, no differences were found in depressive symptoms $t=-0.02$, $p=.845, \eta^{2}=0.000$ (MBPI group $M=11.02 ; S D=7.05$; control group $M=11.35 ; S D=9.27$ ) and in infertility selfefficacy $t=0.83, p=.411, \eta^{2}=0.008$ (MBPI group $M=81.87 ; S D=24.45$; control group $M=86.35 ; S D=26.98$ ).

Figure 1 graphically displays participants' score changes in self-efficacy and depression from pre-treatment (T1) to posttreatment (T2) in the MBPI and control groups.

Scores on both measures were statistically different between the two groups, $F(1,90)=13.875, p<.001$, $\eta_{p}^{2}=0.13$ and $F(1,90)=8.064, p=.006, \eta_{p}{ }^{2}=0.08$, for self-efficacy and depressive symptoms scores, respectively.

A mediation analysis was conducted to examine whether changes in self-efficacy mediated the effect of the MBPI intervention in reducing depressive symptoms (Fig. 2).

Straight lines represent the direct effects. The dotted line represents the indirect effect from the bootstrap analysis. BC 95\% CI denotes bias-corrected 95\% confidence interval; infertility self-efficacy and depressive symptom scores at T1 were included in the model as covariates.

The results showed that the mediation effect was significant (estimate: 1.61, bootstrap bias-corrected $95 \%$ confidence
Table 1 Participant descriptive characteristics and group comparisons on demographic and clinical variables

\begin{tabular}{|c|c|c|c|c|c|c|c|}
\hline 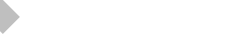 & \multicolumn{2}{|c|}{ MBPI group $(n=55)$} & \multicolumn{2}{|c|}{ Control group $(n=37)$} & \multirow[t]{2}{*}{$t(90)$} & \multirow[t]{2}{*}{$p$} & \multirow[t]{2}{*}{ Cohen's $d$} \\
\hline & $M$ & $S D$ & $M$ & $S D$ & & & \\
\hline Age & 34.87 & 4.20 & 33.14 & 3.94 & 1.20 & 0.049 & 0.42 \\
\hline Years of education & 15.82 & 2.26 & 14.84 & 3.48 & 1.64 & 0.105 & 0.33 \\
\hline Years of marriage & 6.62 & 3.57 & 5.70 & 3.25 & 1.25 & 0.215 & 0.27 \\
\hline \multirow[t]{2}{*}{ Infertility duration } & 3.35 & 2.53 & 3.05 & 2.44 & 0.55 & 0.584 & 0.12 \\
\hline & $N$ & $\%$ & $N$ & $\%$ & $X^{2 \mathrm{a}}$ & $p$ & Cramer's $V$ \\
\hline \multicolumn{8}{|l|}{ Infertility factor } \\
\hline Female & 19 & 34.50 & 15 & 40.50 & & & \\
\hline Male & 12 & 21.80 & 14 & 37.80 & 7.07 & 0.072 & 0.22 \\
\hline Female and male & 14 & 25.50 & 7 & 18.90 & & & \\
\hline Unknown & 10 & 18.20 & 1 & 2.70 & & & \\
\hline
\end{tabular}

${ }^{\text {a }}$ Chi-square test with Monte Carlo simulation 

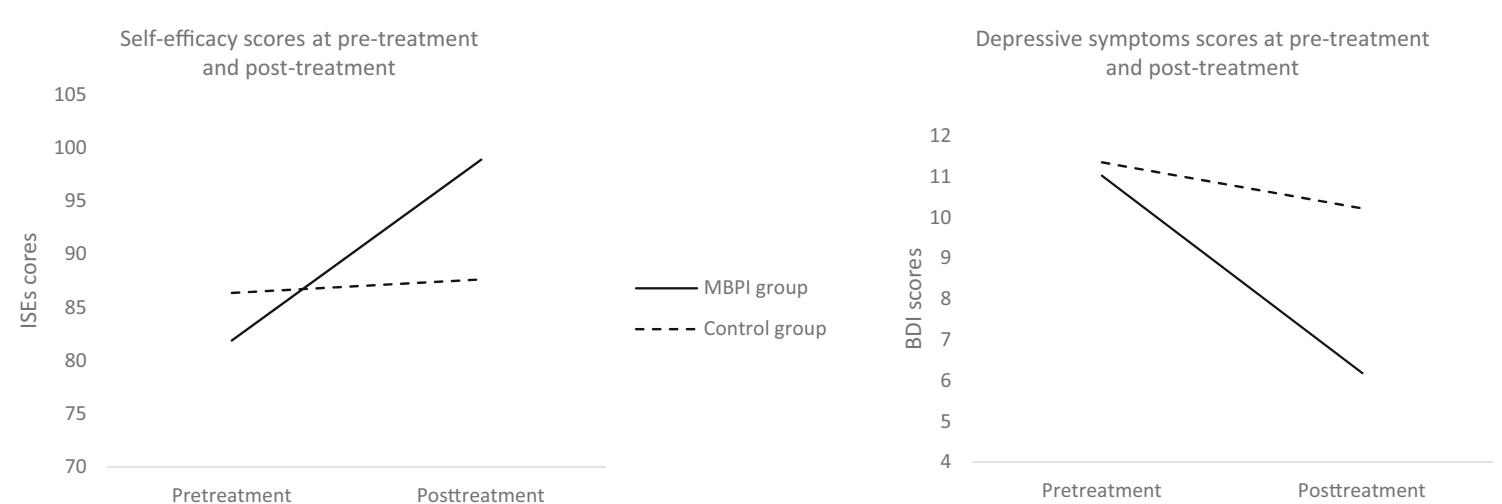

Fig. 1 Changes in self-efficacy and depressive symptoms scores from pre-treatment to post-treatment in MBPI and control groups

interval $0.56 ; 3.47$ ), that is, the MBPI increased infertility selfefficacy, which was in turn associated with a reduction of the depressive symptoms.

The effect of the condition (control vs. intervention) in the self-efficacy score at T2 (path $a$ ), while controlling for selfefficacy and depressive symptoms at $\mathrm{T} 1$, was 0.57 $(\mathrm{SE}=0.08)$, with $p<.001$, which indicates that being in the intervention group was associated with an increase in selfefficacy after the intervention. When testing path $b$, that is, the effect of self-efficacy in depressive scores while controlling for self-efficacy and depressive symptoms at $\mathrm{T} 1$, the effect was $0.13(\mathrm{SE}=0.03)$, with $p<.001$, showing that selfefficacy at T2 was negatively associated with depressive symptoms. The test of the $a b$ path, that is, the indirect effect of the condition on depressive symptoms at $\mathrm{T} 2$, was significant (estimate: 1.61 , bootstrap bias-corrected $95 \%$ confidence interval $0.56 ; 3.47)$. According to this result, the MBPI increased infertility self-efficacy, which was in turn associated with a reduction of depressive symptoms.

\section{Discussion}

The aim of the current study was to examine the mechanism underlying the reduction of depressive symptoms in women facing infertility after completing the MBPI (Galhardo et al. 2013b). More specifically, in this study we tested whether the change in depressive symptoms could be explained by the increase in the perception of self-efficacy regarding infertility. Indeed, the results showed that women facing infertility who received the MBPI intervention increased their perceptions of self-efficacy in dealing with infertility, which in turn decreased their depressive symptoms.

Depressive symptoms are related to biased thoughts about oneself, the world, and the future, and contents of worthlessness and self-blame are rather recurrent (Williams et al. 2007). With mindfulness practice, participants became more eonseious of their internal and external experience in the present moment with an attitude of openness and curiosity (KabatZinn 1990). Therefore, painful thoughts (e.g., "I will never be a mum," "What if this IVF treatment fails?") and feelings (e.g., anxiety, sadness, anger) related to the past or to the future are recognized without trying to suppress or modify them and this may lead to a decrease of the impact of these private events (Williams et al. 2007). By increasing the perception of oneself as being able to cope with painful experiences such as infertility, these women might present a more positive emotional state, persist with medical treatment, or achieve a family-building resolution other than the one with biological children (Cousineau et al. 2006). In fact, women showing higher levels of self-efficacy tend to look at demanding tasks, such as dealing with infertility, from a standpoint of challenge rather than of threat, set significant goals, and commit to accomplishing them (Yong 2010). Similar results were found by Zhang and Jin (2016) showing that self-efficacy partially mediated the relationship between social support and postpartum depression. This mediation role of self-efficacy was also found in other health conditions such as spinal cord injury, with self-efficacy mediating the impact of chronic pain on mood (Craig et al. 2013), and fibromyalgia, with selfefficacy as a mediator between self-reported pain and functional limitation and depression (Peñacoba et al. 2015).
Fig. 2 Intervention effects of the MBPI in reduction of depressive symptoms in women with infertility

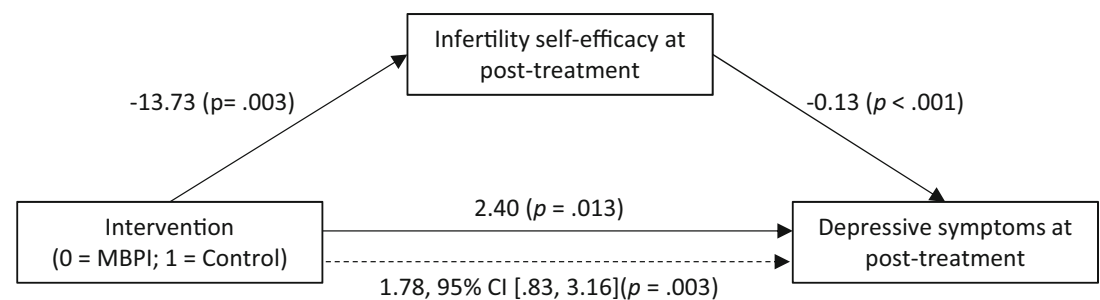


Our findings demonstrated that one potential mechanism by which the MBPI is effective in reducing depressive symptoms in infertile patients is by increasing the patients' confidence levels on aspects of cognitive, emotional, and behavioral skills related to infertility. These findings should be interpreted takinto account some limitations. This was not a randomized control trial and was not a blind study given the nature of practical impediments to collecting the sample and the fact that double-blind placebo-controlled trials are not feasible for mindfulness interventions (Davidson and Kaszniak 2015). Participants were allocated to the MBPI condition and to the control group based on geographical criteria; nevertheless, except for age, no significant differences were found in demographic characteristics and the groups were equivalents at baseline concerning the psychological variables addressed and were being medically assisted in the same clinics, reducing the probability of eventual effects of geographical differences. Participants' recruitment was conducted via the Internet, which means that they have Internet access and may be particularly informed and motivated to some kind of psychological intervention. In addition, the findings clarify one of the mechanisms by which the MBPI exerted its effect, but other processes may have occurred contributing to its efficacy. In fact, the overlap across measures of self-efficacy, acceptance, and mindfulness has been previously suggested (Turner et al. 2016) and this may also happen in the current study. Finally, the study just included two waves of data, assuming stationary effects between the mediator and the outcomes, which may introduce some bias (Cole and Maxwell 2003).

Despite the limitations mentioned, the current study allows a better understanding of the mediator effect of self-efficacy on depressive symptoms and highlights the importance of promoting self-efficacy. This points to the relevance of specif ieally addressing the way women dealing with infertility perceive their abilities to face this condition and the strains of medical treatment when designing psychological intervention programs.

Acknowledgements We thank all the participants in the study for their contribution. The authors also like to thank the national patients association for the support provided.

Author Contributions AG: designed and executed the study, assisted with the data analyses, and wrote the paper. MMR: analyzed the data and wrote the paper. MC: collaborated with the design and writing of the study. JPG: collaborated with the design and the discussion of results.

Funding Information This research has been supported by the first author Ph.D. Grant (SFRH/BD/68392/2010), sponsored by FCTPortuguese national funding agency for science, research and technology.

\section{Compliance with Ethical Standards}

Ethical Approval All procedures performed in studies involving human participants were in accordance with the ethical standards of the institutional and/or national research committee and with the 1964
Helsinki Declaration and its later amendments or comparable ethical standards. Ethical approval was obtained from the Scientific Council of the Faculty of Psychology and Educational Sciences of the University of Coimbra.

Conflict of Interest The authors declare that they have no conflict of interest.

Informed Consent Written informed consent was obtained from all individual participants included in the study.

\section{References}

Baer, R. A. (2003). Mindfulness training as a clinical intervention: a conceptual and empirical review. Clinical Psychology: Science and Practice, 10(2), 125-143.

Bandura, A. (1994). Self-efficacy. In Ramachaudran (Ed.), Encyclopedia of human behavior (Vol. 4, pp. 71-81). New York: Academic Press.

Beck, A., Ward, C., Mendelson, M., Mock, J., \& Erbaugh, J. (1961). An inventory for measuring depression. Archives of General Psychiatry, 4, 561-571.

Chen, T. H., Chang, S. P., Tsai, C. F., \& Juang, K. D. (2004). Prevalence of depressive and anxiety disorders in an assisted reproductive technique clinic. Human Reproduction, 19(10), 2313-2318.

Cole, D. A., \& Maxwell, S. E. (2003). Testing mediational models with longitudinal data: questions and tips in the use of structural equation modeling. Journal of Abnormal Psychology, 112(4), 558-577. https://doi.org/10.1037/0021-843X.112.4.55.

Cousineau, T. M., Green, T. C., Corsini, E. A., Barnard, T., Seibring, A. R., \& Domar, A. D. (2006). Development and validation of the infertility self-efficacy scale. Fertility and Sterility, 85(6), 16841696. https://doi.org/10.1016/j.fertnstert.2005.10.077.

Covington, S. N., \& Adamson, G. D. (2015). Collaborative reproductive healthcare model. In S. N. Covington (Ed.), Fertility counseling: clinical guide and case studies (pp. 1-32). Cambridge: Cambridge University Press.

Craig, A., Tran, Y., Siddall, P., Wijesuriya, N., Lovas, J., Bartrop, R., \& Middleton, J. (2013). Developing a model of associations between chronic pain, depressive mood, chronic fatigue, and self-efficacy in people with spinal cord injury. The Journal of Pain, 14(9), 911-920. https://doi.org/10.1016/j.jpain.2013.03.002.

Datta, J., Palmer, M. J., Tanton, C., Gibson, L. J., Jones, K. G., Macdowall, W., ... Wellings, K. (2016). Prevalence of infertility and help seeking among 15000 women and men. Human Reproduction, 31(9). https://doi.org/10.1093/humrep/dew123.

Davidson, R. J., \& Kaszniak, A. W. (2015). Conceptual and methodological issues in research on mindfulness and meditation. The American Psychologist, 70(7), 581-592. https://doi.org/10.1037/a0039512.

Domar, A. D., Clapp, D., Slawsby, E. A., Dusek, J., Kessel, B., \& Freizinger, M. (2000). Impact of group psychological interventions on pregnancy rates in infertile women. Fertility and Sterility, 73(4), 805-811.

Domar, A. D., Seibel, M. M., \& Benson, H. (1990). The mind/body program for infertility: a new behavioral treatment approach for women with infertility. Fertility and Sterility, 53(2), 246-249.

Eugster, A., \& Vingerhoets, A. J. J. M. (1999). Psychological aspects of in vitro fertilization: a review. Social Science and Medicine, 48, 575-589.

Faramarzi, M., Pasha, H., Esmailzadeh, S., Kheirkhah, F., Hajian-Tilaki, K., \& Salmalian, H. (2014). A survey of correlation infertility selfefficacy with behavioral health scales in infertile women. Health, 6 , 943-949. https://doi.org/10.4236/health.2014.610119. 
Fu, B., Yan, P., Yin, H., Zhu, S., Liu, Q., Liu, Y., Dai, C., Tang, G., Yan, C., Lei, J. (2016). Psychometric properties of the Chinese version of the Infertility Self-Efficacy Scale. International Journal of Nursing Sciences, 3(3), 259-267. https://doi.org/10.1016/j.ijnss.2016.07. 008.

Galhardo, A., Cunha, M., \& Pinto-Gouveia, J. (2013a). Measuring selfefficacy to deal with infertility: psychometric properties and confirmatory factor analysis of the Portuguese version of the infertility self-efficacy scale. Research in Nursing and Health, 36, 65-74. https://doi.org/10.1002/nur.21516.

Galhardo, A., Cunha, M., \& Pinto-Gouveia, J. (2013b). Mindfulnessbased program for infertility: efficacy study. Fertility and Sterility, 100(4), 1059-1067. https://doi.org/10.1016/j.fertnstert.2013.05. 036.

Galhardo, A., Cunha, M., \& Pinto-Gouveia, J. (2016). Acceptability and feasibility of the mindfulness based program for infertility (PBMI). European Psychiatry, 33(Supplement), S519. https://doi.org/10. 1016/j.eurpsy.2016.01.1918.

Gandoy-Crego, M., Clemente, M., Gomez-Cantorna, C., GonzalezRodriguez, R., \& Reig-Botella, A. (2016). Self-efficacy and health: the SEH scale. American Journal of Health Psychology, 40(3), 389395. https://doi.org/10.5993/AJHB.40.3.11.

Gotink, R. A., Chu, P., Busschbach, J. J., Benson, H., Fricchione, G. L., \& Hunink, M. G. (2015). Standardised mindfulness-based interventions in healthcare: an overview of systematic reviews and metaanalyses of RCTs. PloS One, 10(4), e0124344. https://doi.org/10. 1371/journal.pone.0124344.

Greil, A. L. (1997). Infertility and psychological distress: a critical review of the literature. Social Science and Medicine, 45(11), 1679-1704.

Harris, R. (2009). ACT made simple: a quick-start guide to ACT basics and beyond. Oakland: New Harbinger Publications, Inc.

Hayes, A. (2013). Introduction to mediation, moderation, and conditional process analysis: a regression based approach. New York: Guilford.

Hayes, A. F., \& Rockwood, N. J. (2016). Regression-based statistical mediation and moderation analysis in clinical research: observations, recommendations, and implementation. Behavior Research Therapy. https://doi.org/10.1016/j.brat.2016.11.001.

Hayes, S. C., Strosahl, K., \& Wilson, K. G. (1999). Acceptance and commitment therapy: an experiential approach to behavior change. New York: Guilford Press.

Hou, R. J., Wong, S. Y. S., Yip, B. H. K., Hung, A. T. F., Lo, H. H. M., Chan, P. H. S., ... Ma, S. H. (2014). The effects of MindfulnessBased Stress Reduction Program on the mental health of family caregivers: a randomized controlled trial. Psychotherapy and Psychosomatics, 83(1), 45-53.

Kabat-Zinn, J. (1990). Full catastrophe living. New York: Delta.

Luberto, C. M., Cotton, S., McLeish, A. C., Mingione, C. J., \& O'Bryan, E. M. (2014). Mindfulness skills and emotion regulation: the mediating role of coping self-efficacy. Mindfulness, 5(4), 373-380. https://doi.org/10.1007/s12671-012-0190-6.

Mackinnon, D. P., Lockwood, C. M., \& Williams, J. (2004). Confidence limits for the indirect effect: distribution of the product and resampling methods. Multivariate Behavioral Research, 39(1), 99. https:// doi.org/10.1207/s15327906mbr3901 4.

Mascarenhas, M. N., Flaxman, S. R., Boerma, T., Vanderpoel, S., \& Stevens, G. A. (2012). National, regional, and global trends in infertility prevalence since 1990: a systematic analysis of 277 health surveys. PLoS Medicine, 9(12), e1001356. https://doi.org/10.1371/ journal.pmed.1001356.

Moura-Ramos, M., Gameiro, S., Soares, I., Almeida-Santos, T., \& Canavarro, M. C. (2010). Psychosocial adjustment in infertility: a comparison study of infertile couples, couples undergoing assited reproductive technologies and presumed fertile couples. Psicologia, Saúde \& Doenças, 11(2), 299-319.

Peñacoba, C. P., Velasco, L. F., Écija, C. G., Cigarán, M. M., Bedmar, D. C., \& Fernández-de-las-Peñas, C. (2015). Self-efficacy and affect as mediators between pain dimensions and emotional symptoms and functional limitation in women with fibromyalgia. Pain Management Nursing, 16(1), 60-68. https://doi.org/10.1016/j.pmn. 2014.04.005.

Sejbaek, C. S., Hageman, I., Pinborg, A., Hougaard, C. O., \& Schmidt, L. (2013). Incidence of depression and influence of depression on the number of treatment cycles and births in a national cohort of 42880 women treated with ART. Human Reproduction, 28(4), 1100-1109. https://doi.org/10.1093/humrep/des442.

Turner, J. A., Anderson, M. L., Balderson, B. H., Cook, A. J., Sherman, K. J., \& Cherkin, D. C. (2016). Mindfulness-based stress reduction and cognitive behavioral therapy for chronic low back pain: similar effects on mindfulness, catastrophizing, self-efficacy, and acceptance in a randomized controlled trial. Pain, 157(11), 2434-2444. https://doi.org/10.1097/j.pain.0000000000000635.

Vaz Serra, A., \& Abreu, J. (1973). Aferição dos quadros clínicos depressivos: Ensaio de aplicação do Inventário Depressivo de Beck a uma amostra de doentes deprimidos. Coimbra Médica, 20, 623-644

Verhaak, C. M., Lintsen, A. M., Evers, A. W., \& Braat, D. D. (2010). Who is at risk of emotional problems and how do you know? Screening of women going for IVF treatment. Human Reproduction, 25(5), 1234-1240. https://doi.org/10.1093/humrep/ deq054.

Verhaak, C. M., Smeenk, J. M., Evers, A. W., Kremer, J. A., Kraaimaat, F. W., \& Braat, D. D. (2007). Women's emotional adjustment to IVF: a systematic review of 25 years of research. Human Reproduction Update, 13(1), 27-36. https://doi.org/10.1093/humupd/dml040.

Volgsten, H., Skoog, S. A., Ekselius, L., Lundkvist, O., \& Sundstrom, P. I. (2010). Risk factors for psychiatric disorders in infertile women and men undergoing in vitro fertilization treatment. Fertility and Sterility, 93(4), 1088-1096. https://doi.org/10.1016/j.fertnstert. 2008.11.008.

Williams, M., Teasdale, J., Segal, Z., \& Kabat-Zinn, J. (2007). The mindfulness way through depression. New York: The Guilford Press.

Yong, F. L. (2010). A study on the self-efficacy and expectancy for success of pre-university students. European Journal of Social Sciences, 13(4), 514-524.

Zegers-Hochschild, F., Adamson, G. D., de Mouzon, J., Ishihara, O., Mansour, R., Nygren, K., et al. (2009). International Committee for Monitoring Assisted Reproductive Technology (ICMART) and the World Health Organization (WHO) revised glossary of ART terminology, 2009. Fertility and Sterility, 92(5), 15201524. https://doi.org/10.1016/j.fertnstert.2009.09.009.

Zhang, Y., \& Jin, S. (2016). The impact of social support on postpartum depression: the mediator role of self-efficacy. Journal of Health Psychology, 21(5), 720-726. https://doi.org/10.1177/ 1359105314536454. 